\title{
ÉVOLUTION SPATIO-TEMPORELLE DE LA CONTRIBUTION DU REPEUPLEMENT EN TRUITE (SALMO TRUTTA L.) RÉALISÉ A DES STADES PRÉCOCES DANS LE BASSIN AMONT DU FIER (74).
}

A. CAUDRON (1), A. CHAMPIGNEULLE (2)

(1) Fédération de Haute-Savoie pour la Pêche et la Protection du Milieu Aquatique, 1 rue de l'Industrie, 74000 ANNECY, France.

(2) INRA, Station d'Hydrobiologie Lacustre, BP 511, 74203 THONON Cedex, France.

\section{RÉSUMÉ}

Les pratiques de repeuplement en truite à des stades précoces (alevins nourris et truitelles $3-5 \mathrm{~cm}$ ) ont été évaluées par la technique de fluoromarquage des otolithes sur le bassin amont du Fier pour trois cohortes (1995, 1996, 1997).

La contribution du repeuplement et du recrutement naturel a été suivie à la fois dans la population en place et dans les captures par pêche à la ligne.

Au stade $0+$ en place à l'automne, la contribution des poissons alevinés est relativement importante pour les trois cohortes considérées. Cependant, l'analyse du rapport entre le nombre d'alevins introduits au printemps et le nombre de truitelles recapturées à l'automne fait apparaître un taux d'implantation très faible des $0+$ marqués quelle que soit la densité alevinée. La contribution des marqués chute brutalement au stade 1+ en place (cohorte 95 et 96) et devient très faible. Cette tendance se poursuit au stade 2+ en place pour la cohorte 95 avec des taux de marqués qui diminuent encore.

Dans les captures par pêche à la ligne le taux de 2+ marqués est supérieur à celui observé dans la population en place à l'automne mais la contribution du recrutement naturel reste toujours dominante. Ensuite, la contribution de repeuplement suit la même dynamique que dans la population en place puisqu'il diminue avec l'âge. Elle devient négligeable au stade $3+$ et nulle à partir de $4+$.

Mots-clés : Truite fario, repeuplement, marquage, otolithes, pêche à la ligne. 


\title{
SPATIO-TEMPORAL EVOLUTION OF THE CONTRIBUTION OF BROWN TROUT (SALMO TRUTTA L.) RESTOCKING REALISED AT PRECOCIOUS STAGES IN THE UPSTREAM PART OF THE RIVER FIER BASIN.
}

\begin{abstract}
The technique of fluoromarking otoliths has been used to evaluate the practices of brown trout (Salmo trutta L.) stocking at precocious stages (fed fry and 3-5 cm trouts long), in the upstream part of the river Fier, with regards to three cohorts $(1995,1996,1997)$.

The stocking and the natural recruitment contribution has been observed through the standing population and through the angling catches.

For each of the three cohorts, there is a rather high contribution of the stocked fry at the autumnal $0+$ standing population. However, the ratio between the initially stocked fry and the recaptured ones at the automn reveals a relatively low implantation rates, whatever the stocked fry density has been. The contribution of the stocked fry in the 1+ standing population $(1995,1996$ cohorts) brutally decrease, down to a low value. For the 2+ standing population (1995 cohort), this contribution is even lower.

The angling catches show a higher rate of $2+$ marked fishes compared to the rate of marked trout in the standing population but the natural recruitment contribution is always prevailing. At later stages, the stocking contribution decreases with the age. It becomes negligible at the $3+$ stage and vanishes at the $4+$ stage.
\end{abstract}

Key-words : brown trout, restocking, marking, otoliths, angling.

\section{INTRODUCTION}

Depuis le milieu du XIX ${ }^{\text {ème }}$ siècle, des opérations régulières d'empoissonnements en truite commune (Salmo trutta L.) sont pratiquées en France en vue de compenser la diminution du potentiel de recrutement naturel liée aux altérations de l'habitat et pour tenter de répondre à la demande des pêcheurs (THIBAULT, 1983). En effet, l'objectif principal des repeuplements pratiqués par les gestionnaires est d'aboutir au maintien et/ou à une augmentation sensible du stock de poissons adultes capturables par pêche à la ligne.

Plusieurs études traitent de l'impact, en particulier génétique, de ces repeuplements sur les populations sauvages (GUYOMARD, 1991 ; POTEAUX et BERREBI, 1997) mais peu (BEAUDOU, 1993 ; CHAMPIGNEULLE et al., 1990) s'intéressent à l'efficacité proprement dite de ces pratiques en terme de quantification de leur contribution aux populations en place et aux captures par la pêche de loisir.

Les principaux travaux publiés sur ce sujet jusqu'à récemment proposaient un suivi de la contribution des poissons de repeuplement uniquement à des stades jeunes $(0+$ et/ou 1+), à des échelles d'étude géographiquement restreintes et avec des quantités de poissons suivis faibles car limitées par les possibilités de marquage existantes (CRESSWELL, 1981).

Le développement de nouvelles techniques de fluoromarquages des otolithes sur les salmonidés (TSUKAMOTO et al., 1989 ; ROJAS BELTRAN et al., 1995a, b ; CHAMPIGNEULLE et ROJAS BELTRAN, 2001) ont permis d'entreprendre des suivis de grandes quantités de poissons alevinés à des stades précoces. Des études ont été réalisées dans ce sens conjointement sur le Doubs (DEGORGI et CHAMPIGNEULLE, 2000 ) et sur le Fier amont. Les résultats obtenus sur le Fier sont l'objet de cette présente publication. 
L'approche expérimentale mise en place avait pour objectifs d'évaluer au sein d'un sous-bassin hydrographique l'efficacité des méthodes de repeuplement telles qu'elles sont pratiquées actuellement sur ce site par les gestionnaires de la pêche. Pour cela, l'évolution de la dynamique de la contribution du repeuplement a été suivie à différents stades de développement (juvéniles et adultes) dans la population en place durant trois années consécutives et dans les captures des pêcheurs pendant les trois autres années suivantes. Ce travail a été entrepris à une large échelle spatiale puisque l'efficacité du repeuplement a été suivi sur les $35 \mathrm{~km}$ du réseau hydrographique amont du Fier comprenant la rivière principale et deux affluents.

\section{MATÉRIEL ET MÉTHODES}

\section{Localisation de l'étude}

Le Fier est l'un des principaux cours d'eau de Haute-Savoie. II prend sa source à $2011 \mathrm{~m}$ d'altitude au pied du Mont Charvin dans le massif des Aravis et se jette en rive gauche du Rhône à l'aval de Seyssel. Son cours long de $69 \mathrm{~km}$ est orienté Est-Ouest et draine un bassin versant d'une superficie de $1386 \mathrm{~km}^{2}$ (Figure 1).

La zone d'étude regroupe le Fier et deux de ses principaux affluents situés à proximité de Thônes, le Nom et le Chamfroid (Figure 1).

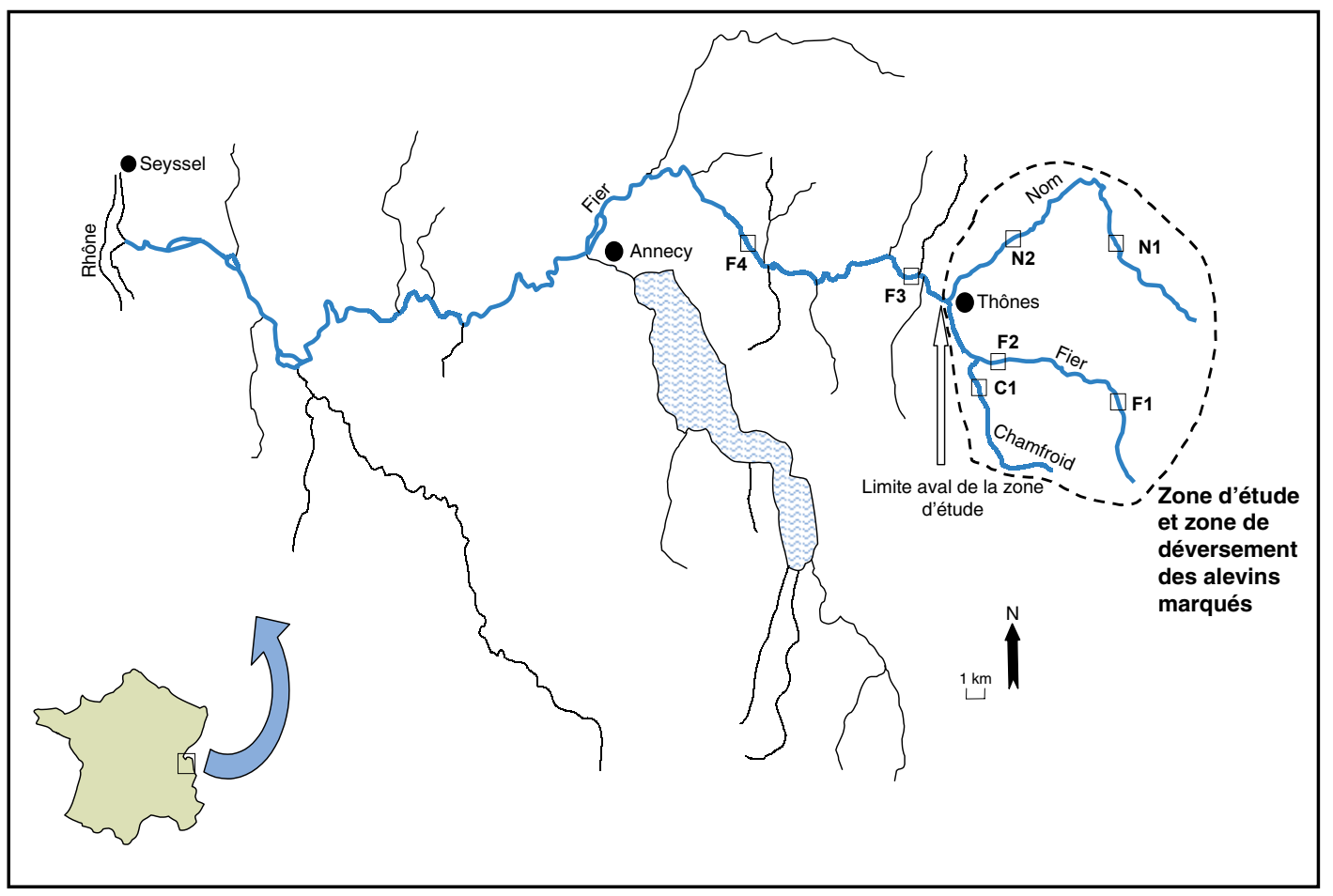

\section{Figure 1}

Présentation du bassin du Fier, localisation de la zone d'étude et de déversement des alevins marqués et des stations d'échantillonnages.

\section{Figure 1}

Map of the River Fier basin, localization of the upstream zone under study and used for the stocking of marked fry and the sampling stations. 
Le Nom, d'une longueur de $17 \mathrm{~km}$ pour un bassin versant de $73 \mathrm{~km}^{2}$, se jette dans le Fier à Thônes. Le Chamfroid, lui, conflue avec le cours principal à l'amont de Thônes après avoir parcouru $7 \mathrm{~km}$. La limite aval de cette zone d'étude est matérialisée par un seuil artificiel constituant un obstacle infranchissable uniquement à la montaison et quelles que soient les conditions de débit.

Dans le secteur étudié, le Fier et ses affluents sont classés en première catégorie piscicole et leur peuplement pisciaire est constitué pour l'essentiel par la truite commune (Salmo trutta) et le chabot (Cottus gobio). Les pêches électriques d'inventaire réalisées sur la zone d'étude ont montré des densités totales de truites variant de 15 à 70 individus $/ 100 \mathrm{~m}^{2}$ et des biomasses totales de truites comprises entre 50 et $150 \mathrm{~kg} / \mathrm{ha}$ (RAYMOND, comm. pers.). Le Fier et ses affluents sur la zone concernée sont des torrents à fond de blocs et galets présentant un régime hydrologique de type nival à influence secondaire pluviale. Leur bassin versant, en dehors des zones urbanisées, est de type agricole et boisé.

\section{Protocole de l'étude}

\section{Marquage des poissons de repeuplement}

En 1995, 1996 et 1997, l'ensemble des truitelles (40 000 à 60 000/an) destinées à être alevinées dans le réseau hydrographique du Fier à l'amont de Thônes ont été marquées par fluoromarquage des otolithes (Tableau I). L'ensemble des poissons alevinés est issu d'une souche domestique d'origine génétique atlantique au niveau de l'ADN mitochodrial (BAUMANN, 1999).

Chaque année, les repeuplements ont été réalisés de manière à répartir la totalité des truitelles de façon homogène sur l'ensemble du linéaire (cours principal et affluents) concerné par la zone d'étude. Ceci correspond à des mises en charge variant principalement entre 13 et 30 individus $/ 100 \mathrm{~m}^{2}$.

Ces marquages ont été réalisés à la tétracycline en 1995-96 et à l'alizarine redS en 1997 selon les techniques décrites respectivement par ROJAS BELTRAN et al. (1995b) et CHAMPIGNEULLE et ROJAS BELTRAN (2001). Les alevinages sur la zone concernée ont été effectués par les gardes pisciculteurs de l'AAPPMA « Annecy-Rivières 》 au stade alevins nourris et surtout truitelle, entre 3 et $5 \mathrm{~cm}$. Les poissons ont été introduits au mois de juin de chaque année après la fonte des neiges afin d'assurer des conditions hydrologiques favorables et stables.

Des lots témoins, marqués avec les techniques utilisées, gardés en pisciculture ont permis de vérifier la bonne prise du marquage ainsi que la pérennité de la marque : $100 \%$ encore marqués à 4 ans.

Le reste du bassin versant du Fier est régulièrement repeuplé en alevins non marqués. Cependant, le seuil infranchissable marquant la limite aval de la zone d'étude évite toute remontée de ces poissons qui pourraient invalider nos résultats.

\section{Echantillonnage par pêche électrique}

Des pêches électriques d'inventaire (méthode de DE LURY: 2 passages consécutifs) ou de sondage (1 seul passage) ont eu lieu en automne de chaque année sur 5 stations de la zone de déversement réparties de la façon suivante : F1 et F2 respectivement en amont et en aval du Fier, N1 et N2 respectivement en amont et en aval du Nom et C1 en aval du Chamfroid (Tableau II, Figure 1). 
Tableau I

Quantités alevinées et répartition des alevins marqués sur la zone d'étude en 1995, 96 et 97.

Table I

Stocking data and repartition of stocked marked fish in the study zone for 1995, 96 and 97.

\begin{tabular}{|c|c|c|c|c|c|c|c|c|c|c|c|}
\hline \multicolumn{3}{|c|}{ Zone d'étude } & \multicolumn{2}{|c|}{ Année 1995} & \multicolumn{2}{|c|}{ Année 1996} & \multicolumn{2}{|c|}{ Année 1997} & \multicolumn{2}{|c|}{ Total des 3 années } & \multirow[b]{2}{*}{ TOTAL } \\
\hline Sous bassin & Cours d'eau & $\begin{array}{l}\text { Longueur } \\
\text { en km }\end{array}$ & $\begin{array}{c}\text { Stade alevins } \\
\text { nourris }\end{array}$ & $\begin{array}{l}\text { Stade truitelle } \\
(3-5 \mathrm{~cm})\end{array}$ & $\begin{array}{c}\text { Stade alevins } \\
\text { nourris }\end{array}$ & $\begin{array}{l}\text { Stade truitelle } \\
(3-5 \mathrm{~cm})\end{array}$ & $\begin{array}{c}\text { Stade alevins } \\
\text { nourris }\end{array}$ & $\begin{array}{c}\text { Stade truitelle } \\
(3-5 \mathrm{~cm})\end{array}$ & $\begin{array}{c}\text { Stade alevins } \\
\text { nourris }\end{array}$ & $\begin{array}{c}\text { Stade truitelle } \\
(3-5 \mathrm{~cm})\end{array}$ & \\
\hline Fier & Le Fier & 12 & & 23000 & 6230 & 16770 & 6230 & 16770 & 12460 & 56540 & 69000 \\
\hline \multirow{3}{*}{ Nom } & Le Nom & 13 & & 14200 & & 21900 & & 18050 & & 54150 & 54150 \\
\hline & $\begin{array}{l}\text { Ruisseau des } \\
\text { Prises }\end{array}$ & 2 & & 2200 & & 2800 & & 2500 & & 7500 & 15000 \\
\hline & $\begin{array}{l}\text { Ruisseau de } \\
\text { Merdassier }\end{array}$ & 0,5 & & 600 & & 700 & & 650 & & 1950 & 1950 \\
\hline \multirow{3}{*}{ Chamfroid } & Le Chamfroid & 4,5 & & 3000 & 3000 & & 3000 & & 6000 & 3000 & 9000 \\
\hline & $\begin{array}{l}\text { Ruisseau des } \\
\text { Grangettes }\end{array}$ & 1 & & 700 & 700 & & 700 & & 1400 & 700 & 2100 \\
\hline & $\begin{array}{l}\text { Ruisseau de } \\
\text { Belchamps }\end{array}$ & 0,5 & & 300 & 300 & & 300 & & 600 & 300 & 900 \\
\hline \multicolumn{2}{|c|}{ TOTAL } & 33,5 & & 44000 & 10230 & 42170 & 10230 & 37970 & 20460 & 124140 & 152100 \\
\hline
\end{tabular}

Tableau II

Principales caractéristiques des stations d'échantillonnage et densité alevinée sur celles-ci pour la période 1995-97.

\section{Table II}

Main sampling stations characteristics and stocking densities for the given stations within the period 1995-1997.

\begin{tabular}{|c|c|c|c|c|c|c|c|c|}
\hline \multirow{2}{*}{ Station } & \multirow{2}{*}{$\begin{array}{l}\text { Largeur moyenne de la } \\
\text { station (en } \mathrm{m} \text { ) }\end{array}$} & \multirow{2}{*}{ Surface $\left(\mathrm{m}^{2}\right)$} & \multicolumn{3}{|c|}{ Habitats (en \%) } & \multicolumn{3}{|c|}{ Densité alevinée / $100 \mathrm{~m}^{2}$} \\
\hline & & & Courant & Plat & Profond $(>0,60 \mathrm{~cm})$ & 1995 & 1996 & 1997 \\
\hline F1 & 5 & 450 & 90 & 0 & 10 & 30 & 30 & 30 \\
\hline F2 & 9,5 & 874 & 80 & 0 & 20 & 30 & 30 & 30 \\
\hline N1 & 5,5 & 402 & 75 & 5 & 20 & 16 & 24 & 20 \\
\hline N2 & 9,5 & 874 & 80 & 0 & 20 & 16 & 24 & 20 \\
\hline C1 & 6,5 & 670 & 90 & 0 & 10 & 13 & 13 & 13 \\
\hline
\end{tabular}


Deux stations additionnelles, $\mathrm{F} 3$ et $\mathrm{F} 4$, situées sur le cours de la rivière principale, en aval de la zone de déversement des marqués, ont été également échantillonnées en 1995 et 1996 mais uniquement par sondage pour repérer la présence éventuelle de juvéniles qui auraient dévalé de la zone de déversement en amont.

Pour chacune de ces stations, un échantillon de truites a été sacrifié au hasard dans des gammes de taille susceptibles de contenir les différentes classes d'âge recherchées $(0+, 1+$ et $2+)$. La taille finale des échantillons examinés dans les différentes classes d'âge a résulté des lectures d'âge. En 1995 et 96, les objectifs visés étaient un minimum de $300+$ et de $301+$ par station. Le sacrifice de grands nombres de truites 2+ étant plus difficile à obtenir des gestionnaires, leur échantillonnage a été limité a une cohorte (1995). Par contre des truites d'âge 2+ et supérieur ont été échantillonnées dans la pêche.

\section{Echantillon récolté par les pêcheurs volontaires}

Durant trois autres années consécutives, 1998, 1999 et 2000, des échantillonnages ont été réalisés à l'aide de pêcheurs volontaires qui ont accepté de récolter des données sur leurs captures (date, lieu, taille) et de faire des prélèvements (écailles et tête). Cet échantillonnage s'étend sur l'ensemble des trois saisons de pêche de mars à octobre et porte uniquement sur des poissons capturés à la ligne dépassant la taille légale de $23 \mathrm{~cm}$ (longueur totale). L'effort d'échantillonnage se répartit non seulement sur la zone de déversement des marqués, mais aussi sur l'aval du cours principal du Fier jusqu'à Annecy (Figure 1).

Cette technique d'échantillonnage permet d'une part de suivre l'évolution des cohortes marquées jusqu'au stade adulte et donc de compléter les échantillons prélevés dans la population en place à des stades plus jeunes, et d'autre part de connaître la contribution réelle, dans le panier des pêcheurs, des poissons alevinés plusieurs années auparavant.

\section{Récolte et traitement des données}

\section{Examen des truites}

L'âge de chaque poisson a été estimé par scalimétrie. Le prélèvement d'écailles a été effectué au niveau de la zone optimale définie par OMBREDANE et RICHARD (1990).

Pour chaque truite échantillonnée, la tête est disséquée pour accéder aux sacculi contenant les otolithes (sagittae). Ensuite chaque sagitta (2 par poisson) est collée sur une lame de verre, polie jusqu'au centre, et enfin, observée au microscope Zeiss Axiolab à épifluorescence (lampe à vapeur de mercure HBO 50), pour repérer la présence éventuelle d'une marque.

\section{Tests statistiques utilisés}

Les intervalles de confiance à $95 \%$ des impacts d'alevinage ont été calculés à partir des tables de WILLIAM et BEYER (1986).

La comparaison des distributions des effectifs a été faite à l'aide du Chi carré et du test exact de Fisher (in SPRENT, 1992) dans le cas des petits échantillons. Ces tests statistiques ont été réalisés en utilisant les logiciels STAT ITCF et Excel sous Windows. 


\section{RÉSULTATS}

\section{Contributions du repeuplement et du recrutement naturel pour les cohortes 1995, 1996 et 1997}

Dans la population en place $(0+, 1+, 2+)$ (Tableau III)

L'impact de l'alevinage au stade $0+$ en place en début automne pour les cohortes 95 et 96 , est très faible à fort et présente des variations interstations très importantes. En effet, celles-ci varient de 2,3 à 85,7\% en 1995 et de 11,7 à 75,6 \% en 1996 (Tableau III).

En 1997, de faibles échantillons de 0+ ont été récoltés sur 2 stations lors des pêches électriques (Tableau III). Sur les trois autres stations F1, F2 et N2, les taux de 0+ marqués sont élevés variant de 68,6 à 92,3\% sans différence interstations significative.

Au stade $1+$ en place, l'impact de l'alevinage montre une gamme de variation interstation plus forte pour la cohorte 95 (0 à 63,6 \%) que pour la cohorte 96 (0 à 23,2\%).

Pour la cohorte 95, deux stations, F2 et N2, montrent un effondrement significatif $(p<0,001)$ du taux de marqués avec respectivement $9,6 \%$ et $0 \%$ de poissons alevinés.

La contribution du repeuplement baisse légèrement sur la station $\mathrm{C} 1(5,3 \%)$ et reste faible pour N1 (8\%). Pour ces deux stations, les variations observées ne sont pas significatives (Tableau III).

Pour la cohorte 96, seule la station C1 (0\%) montre un taux de 1+ marqués significativement plus faible que sur les autres stations $(p<0,05)$. Toutes les autres stations ont un impact de l'alevinage au stade $1+$ statistiquement comparable $(p<0,01)$ avec des valeurs comprises entre 14,8 et $23,2 \%$. Un résultat marquant est que la contribution de marqués baisse de façon significative $(p<0,01)$ entre le stade $0+$ et $1+$ pour toutes les stations.

L'impact d'alevinage de la station F1 est beaucoup plus faible pour la cohorte 96 que pour la cohorte 95 puisqu'il passe de $63,6 \%$ à 17,5\%. Sur les autres stations, le taux de $1+$ marqués est identique pour les deux cohortes (Tableau III).

Au stade 2+ (cohorte 1995), les faibles échantillons collectés (sauf pour la station N2) illustrent la difficulté de prélever après la saison de pêche des poissons dont la longueur totale est susceptible d'être supérieure à la taille limite de capture soit $23 \mathrm{~cm}$. Cependant, seules deux stations sur cinq, F1 et N2, contiennent encore des poissons alevinés 2 ans auparavant mais à des taux faibles (respectivement $11,1 \%$ et $3,5 \%$ ). De plus la contribution de l'alevinage mesurée au stade $2+$ sur la station F1 est significativement plus faible $(p<0,05)$ que celle mesurée au stade $1+$ sur cette même station (Tableau III).

Pour la cohorte 1995, toutes les stations de la zone de déversement montrent une diminution importante (voire même une disparition complète pour certaines d'entre elles) du pourcentage de marqués en fonction de l'âge sauf pour la station N1 qui présente une légère augmentation non significative entre le stade 0+ et le stade 1+ (Tableau III).

Sur l'ensemble regroupé des stations de la zone d'étude, le taux de marqués au stade $0+$ est moyen $(39,7 \%)$. II baisse de façon significative $(p<0,001)$ au stade $1+$ et $2+$ ne représentant respectivement plus que 20,4 et $3,1 \%$ de la population en place sur les secteurs échantillonnés. 


\section{Tableau III}

Impact de l'alevinage (en \%) par station et par cohorte sur la zone d'étude dans la population en place et dans la pêche. (n) taille de l'échantillon, (IC $95 \%$ ) indice de confiance à $95 \%$.

\section{Table III}

Percentage of marked fish among different cohorts for the standing population in each station and for samples of angled catches (for the overall study zone). (n) sample size, (IC 95\%) confidence limits at 95's level.

\begin{tabular}{|c|c|c|c|c|c|c|c|}
\hline & \multicolumn{3}{|c|}{ Population automnale en place } & \multicolumn{3}{|c|}{ Captures dans la pêche } \\
\hline & & $\begin{array}{c}0+ \\
\%(\mathrm{n}) \\
(I C 95 \%)\end{array}$ & $\begin{array}{c}1+ \\
\%(\mathrm{n}) \\
(I C 95 \%)\end{array}$ & $\begin{array}{c}2+ \\
\%(\mathrm{n}) \\
(I C 95 \%)\end{array}$ & $\begin{array}{c}2+ \\
\%(\mathrm{n}) \\
(I C 95 \%)\end{array}$ & $\begin{array}{c}3+ \\
\%(n) \\
(I C 95 \%)\end{array}$ & $\begin{array}{c}4 / 5+ \\
\%(\mathrm{n}) \\
(I C 95 \%)\end{array}$ \\
\hline \multirow{6}{*}{ 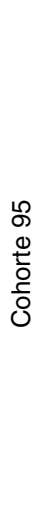 } & $\mathrm{F} 1$ & $\begin{array}{c}85,7 \%(21) \\
(63,7-97)\end{array}$ & $\begin{array}{c}63,6 \%(33) \\
(43,7-78,9)\end{array}$ & $\begin{array}{c}11,1 \%(9) \\
(0,3-48,3)\end{array}$ & $\mathrm{x}$ & $x$ & $\mathrm{x}$ \\
\hline & $\mathrm{F} 2$ & $\begin{array}{l}52,9 \%(34) \\
(35,1-70,2)\end{array}$ & $\begin{array}{l}0 \%(13) \\
(0-24,7)\end{array}$ & $\begin{array}{l}\mathbf{0} \%(9) \\
(0-33,6)\end{array}$ & $x$ & $x$ & $x$ \\
\hline & $\mathrm{N} 1$ & $\begin{array}{c}2,3 \%(44) \\
(0,1-11,5)\end{array}$ & $\begin{array}{c}8 \%(25) \\
(1-27)\end{array}$ & $\begin{array}{l}0 \%(18) \\
(0-18,5)\end{array}$ & $x$ & $x$ & $x$ \\
\hline & N2 & $\begin{array}{c}61,8 \%(55) \\
(49,6-76,2)\end{array}$ & $\begin{array}{l}9,5 \%(52) \\
(3,3-21,8)\end{array}$ & $\begin{array}{l}3,5 \%(57) \\
(0,5-13,2)\end{array}$ & $x$ & $\mathrm{x}$ & $\mathrm{x}$ \\
\hline & C1 & $\begin{array}{l}15 \%(40) \\
(5,6-29,2)\end{array}$ & $\begin{array}{c}5,3 \%(19) \\
(0,1-26)\end{array}$ & $\begin{array}{c}0 \%(3) \\
(0-70,8)\end{array}$ & $X$ & $\mathrm{x}$ & $x$ \\
\hline & Total Zone d'étude & $\begin{array}{c}39,7 \%(194) \\
(37-52)\end{array}$ & $\begin{array}{c}20,4 \%(142) \\
(15,3-30)\end{array}$ & $\begin{array}{c}3,1 \%(96) \\
(0,6-8,3)\end{array}$ & $x$ & $\begin{array}{c}9,5 \%(21) \\
(0-31,7)\end{array}$ & $\begin{array}{l}0 \%(5) \\
(0-8,1)\end{array}$ \\
\hline \multirow{6}{*}{$\begin{array}{l}\mathscr{8} \\
\Phi \\
\Phi \\
\frac{1}{0} \\
\frac{0}{0} \\
0\end{array}$} & $\mathrm{~F} 1$ & $\begin{array}{c}39,7 \%(68) \\
(27,6-52,2)\end{array}$ & $\begin{array}{c}17,5 \%(40) \\
(7-31,4)\end{array}$ & $x$ & $x$ & $x$ & $X$ \\
\hline & F2 & $\begin{array}{c}75,6 \%(119) \\
(63,4-80,8)\end{array}$ & $\begin{array}{c}14,8 \%(27) \\
(4,4-34,9)\end{array}$ & $\mathrm{x}$ & $\mathrm{x}$ & $\mathrm{x}$ & $\mathrm{x}$ \\
\hline & $\mathrm{N} 1$ & $\begin{array}{c}61,7 \%(107) \\
(49,7-69,7)\end{array}$ & $\begin{array}{l}23,2 \%(56) \\
(12,5-35,3)\end{array}$ & $\mathrm{x}$ & $x$ & $x$ & $X$ \\
\hline & N2 & $\begin{array}{c}71,9 \%(32) \\
(51,9-85,8)\end{array}$ & $\begin{array}{c}16,7 \%(18) \\
(3,4-39,6)\end{array}$ & $x$ & $\mathrm{x}$ & $x$ & $x$ \\
\hline & C1 & $\begin{array}{c}11,7 \%(103) \\
(6,9-21,7)\end{array}$ & $\begin{array}{c}0 \%(61) \\
(0-6)\end{array}$ & $\mathrm{x}$ & $x$ & $\mathrm{x}$ & $\mathrm{x}$ \\
\hline & Total Zone d'étude & $\begin{array}{c}50,8 \%(429) \\
(45-55)\end{array}$ & $\begin{array}{c}13,4 \%(202) \\
(7,7-16,4)\end{array}$ & $X$ & $\begin{array}{l}20 \%(45) \\
(9,8-35,3)\end{array}$ & $\begin{array}{c}7,7 \%(13) \\
(0,2-36)\end{array}$ & $\begin{array}{l}0 \%(19) \\
(0-17,6)\end{array}$ \\
\hline \multirow{6}{*}{ 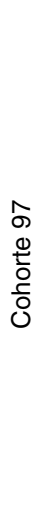 } & $\mathrm{F} 1$ & $\begin{array}{r}68,6 \%(51) \\
(54,1-80,9)\end{array}$ & $\mathrm{x}$ & $x$ & $\mathrm{x}$ & $x$ & $x$ \\
\hline & F2 & $\begin{array}{c}92,3 \%(13) \\
(64-99,8)\end{array}$ & $\mathrm{x}$ & $x$ & $x$ & $x$ & $x$ \\
\hline & $\mathrm{N} 1$ & $\begin{array}{c}50 \%(2) \\
(1,3-98,7)\end{array}$ & $x$ & $x$ & $x$ & $x$ & $\mathrm{x}$ \\
\hline & N2 & $\begin{array}{r}\mathbf{8 8 , 2} \%(17) \\
(63,6-98,5)\end{array}$ & $\mathrm{x}$ & $\mathrm{x}$ & $x$ & $x$ & $x$ \\
\hline & C1 & $\begin{array}{c}100 \%(1) \\
(0-100)\end{array}$ & $\mathrm{x}$ & $x$ & $x$ & $x$ & $x$ \\
\hline & Total Zone d'étude & $\begin{array}{c}76,2 \%(84) \\
(64,1-84)\end{array}$ & $\mathrm{x}$ & $x$ & $\begin{array}{c}11,8 \%(34) \\
(3,3-27,5)\end{array}$ & $\begin{array}{l}0 \%(28) \\
(0-12,3)\end{array}$ & $X$ \\
\hline
\end{tabular}


Pour la cohorte 96, sur l'ensemble regroupé des stations de la zone d'étude, le taux de $0+$ marqués est assez fort $(50,8 \%)$, même légèrement supérieur à celui observé pour la cohorte 95. Au stade 1+, la contribution des poissons alevinés baisse de façon importante et significative $(p<0,001)$ avec seulement $13,4 \%$ de marqués. Le taux de $1+$ marqués de la cohorte 96 est significativement plus faible $(p<0,05)$ que celui de la cohorte 95 (Tableau III).

Pour l'ensemble des 0+ échantillonnés en 1997 sur la zone de déversement, la contribution des marqués est élevée $(76,2 \%)$ et augmente de façon significative $(p<0,001)$ par rapport à la cohorte $95(39,7 \%)$ et $96(50,8 \%)$ (Tableau III).

\section{Dans la pêche $(2+, 3+, \geq 4+)$ (Tableau III)}

Pour les 3 cohortes, la contribution de l'alevinage dans les captures par pêche à la ligne présente une évolution comparable caractérisée par une diminution du taux de marqués avec l'âge (Tableau III). Par exemple pour la cohorte 96, le taux de marqués dans les captures des pêcheurs passe de $20 \%$ à $2+$ à $7,7 \%$ à $3+$ et devient nul à $4+$.

Globalement, la contribution des poissons alevinés dans les captures par pêche à la ligne reste très nettement inférieure à celle du recrutement naturel. Elle devient même nulle à partir du stade 4+ pour les cohorte 95,96 et du stade 3+ pour la cohorte 97.

Cependant, le taux de 2+ marqués dans la pêche pour la cohorte 97 est significativement supérieur $(p<0,05)$ à celui observé dans la population en place pour la cohorte 95 . Pour cette dernière, le taux de marqués parmi les $3+$ pêchés à la ligne $(9,5 \%)$ est légèrement supérieur au taux de marqués parmi les $2+$ de la population automnale en place $(3,1 \%)$. De même, pour la cohorte 96 , le taux de marqués parmi les $2+$ pêchés à la ligne $(20 \%)$ est légèrement supérieur au taux moyen de $1+$ marqués capturés sur la zone d'étude $(13,4 \%)$.

\section{Echantillonnage en aval de la zone de déversement des marqués}

Les résultats des échantillons récoltés par pêche électrique et capture à la ligne en aval de la zone de déversement des marqués sont présentés par classe d'âge dans le Tableau IV.

\section{Tableau IV}

Résultats par classe d'âge des échantillonnages réalisés en aval de la zone d'étude dans la population en place et dans la pêche. (n) taille de l'échantillon, (IC $95 \%$ ) indice de confiance à $95 \%$.

\section{Table IV}

Percent of marked fish among samples realized downstream the studied zone among the standing population and in anglers catches. (n) sample size, (IC 95\%) confidence limits at 95's level.

\begin{tabular}{cccccc} 
& $\begin{array}{c}0+ \\
\%(\mathrm{n}) \\
(I C 95 \%)\end{array}$ & $\begin{array}{c}1+ \\
\%(\mathrm{n}) \\
(I C 95 \%)\end{array}$ & $\begin{array}{c}2+ \\
\%(\mathrm{n}) \\
(I C 95 \%)\end{array}$ & $\begin{array}{c}3+ \\
\%(\mathrm{n}) \\
(I C 95 \%)\end{array}$ & $\begin{array}{c}4+/ 5+ \\
\%(\mathrm{n}) \\
(I C 95 \%)\end{array}$ \\
\hline $\begin{array}{c}\text { Population en place } \\
\text { (F3 + F4) }\end{array}$ & $\begin{array}{c}\mathbf{0} \%(221) \\
(0-1,8)\end{array}$ & $\begin{array}{c}0 \%(13) \\
(0-24,7)\end{array}$ & $\mathrm{X}$ & $\mathrm{X}$ & $\mathrm{X}$ \\
$\begin{array}{c}\text { Captures dans la pêche } \\
\text { C }\end{array}$ & $\mathrm{X}$ & $\mathrm{X}$ & $\begin{array}{c}\mathbf{2 , 7} \%(111) \\
(0,6-8,3)\end{array}$ & $\begin{array}{c}\mathbf{2 , 7} \%(111) \\
(0,6-8,3)\end{array}$ & $\begin{array}{c}0 \%(27) \\
(0-12,3)\end{array}$ \\
\hline
\end{tabular}


Au stade juvénile $0+$ et $1+$ en place à l'automne, aucun individu marqué n'a été trouvé donc aucun ne provenait de la zone de déversement située en amont. Cependant, le nombre de $1+$ échantillonné est faible (13 poissons).

Dans la pêche, le taux de 2+ et $3+$ marqués provenant de la zone de déversement en amont est très faible puisqu'il représente pour les deux classes d'âge 2,7\%. Ces observations montrent cependant qu'une dévalaison des individus marqués a eu lieu même si elle a eu une contribution minime en partie liée à la grande surface de dissolution. nul.

A partir du stade 4+, le taux de marqués provenant de la zone de déversement est

Contribution globale des poissons de repeuplement dans les captures par pêche à la ligne au cours des saisons de pêche 98,99 et 2000

Pour chaque saison de pêche et pour les trois saisons de pêche regroupées, la dynamique de la contribution des poissons alevinés a été étudiée par classe d'âge quelle que soit la cohorte $(95,96,97)$ (Tableau V). Cette démarche permet d'analyser la contribution réelle, directement dans le panier des pêcheurs, des poissons introduits au stade juvénile et du recrutement naturel.

Globalement, la contribution dans la pêche des poissons marqués diminue avec l'âge. Elle est de $20 \%$ pour les $2+$ et de $9,5 \%$ pour les 3+ pour la saison 1998. Une diminution comparable entre ces deux classes d'âge est également observée pour la saison 1999 (Tableau V). En 1999, le nombre de poissons marqués dans les captures quel que soit l'âge représente $10,4 \%$.

\section{Tableau V}

Impact de l'alevinage (en \%) par classe d'âge dans la pêche pour les saisons 1998, 1999, 2000 et pour l'ensemble des saisons réunies. (n) taille de l'échantillon, (IC $95 \%$ ) indice de confiance à $95 \%$.

\section{Table V}

Percentage of marked fish among different age classes in anglers catches for the 1998, 1999, 2000 angling seasons. (n) sample size, (IC 95\%) confidence limits at 95's level.

\begin{tabular}{|c|c|c|c|}
\hline Période & $\begin{array}{c}2+ \\
\%(\mathrm{n}) \\
(I C 95 \%)\end{array}$ & $\begin{array}{c}3+ \\
\%(\mathrm{n}) \\
(I C 95 \%)\end{array}$ & $\begin{array}{c}4+/ 5+ \\
\%(\mathrm{n}) \\
\text { (IC } 95 \%)\end{array}$ \\
\hline 1998 & $\begin{array}{l}20 \%(45) \\
(9,8-35,3)\end{array}$ & $\begin{array}{c}9,5 \%(21) \\
(1,2-30,4)\end{array}$ & $x$ \\
\hline 1999 & $\begin{array}{c}11,8 \%(34) \\
(3,3-27,5)\end{array}$ & $\begin{array}{c}7,7 \%(13) \\
(0,2-36)\end{array}$ & $x$ \\
\hline 2000 & $x$ & $\begin{array}{l}0 \%(28) \\
(0-12,3)\end{array}$ & $\begin{array}{l}0 \%(23) \\
(0-14,2)\end{array}$ \\
\hline Total & $\begin{array}{c}16,5 \%(79) \\
(9,8-28,5)\end{array}$ & $\begin{array}{c}4,8 \%(62) \\
(0,4-11,2)\end{array}$ & $\begin{array}{l}0 \%(24) \\
(0-14,2)\end{array}$ \\
\hline
\end{tabular}


Le taux de 2+ marqués est légèrement supérieur en 1998 (20\%) à celui observé en $1999(11,8 \%)$.

En 2000, le taux de 3+ marqués (0\%) est inférieur à celui observé lors des deux saisons précédentes $(7,7 \%$ en 1999 et $9,5 \%$ en 1998).

Pour les trois saisons de pêche regroupées, la contribution des individus alevinés est déjà faible à l'âge $2+(16,4 \%)$ et continue à diminuer avec l'âge pour représenter seulement $4,8 \%$ à $3+$ et $0 \%$ à partir de $4+$ (Tableau V). Cette baisse du taux des individus marqués est significative entre le stade $2+$ et $3+$ et entre le stade $2+$ et $4+(p<0,05)$.

\section{DISCUSSION}

\section{Dynamique de la contribution du repeuplement dans la population en place et dans la pêche}

Globalement, les résultats montrent que la contribution des poissons alevinés diminue au cours du temps. L'impact d'alevinage au stade $0+$ en place à l'automne est relativement élevé puis s'effondre lorsqu'on le mesure au stade $1+$ et $2+$. Dans la pêche le taux de 2+ marqués est plus important que celui observé dans la population en place. Cependant la contribution du recrutement naturel dans les captures par pêche à la ligne est dans tous les cas dominante et augmente au cours du temps. A partir du stade 4+, la contribution du recrutement naturel est de $100 \%$.

Trois hypothèses peuvent expliquer la contribution importante des poissons de repeuplement à l'âge $0+$ :

a) les alevinages sont effectués en fin de printemps avec des stades précoces (alevins nourris et truitelles) à une période où les paramètres physiques et hydrologiques ( $T^{\circ}$ de l'eau, débit, etc.) sont stables et favorables: la crue de fonte des neiges est achevée, la production d'invertébrés et la température de l'eau sont favorables à la croissance, et les mouvements de juvéniles sont faibles,

b) aux stades auxquels se pratiquent les déversements, l'implantation immédiate des $0+$ dans le milieu peut être favorisée par leur plus grande taille (au moment du déversement) comparativement à ceux issus du recrutement naturel. En effet, l'avantage initial en taille pour les alevins repeuplés est effectivement conservé à l'automne chez les survivants 0+ (CAUDRON, 1998). Cependant le taux de disparition des 0+ alevinés 4 mois après leur déversement est élevé (70 à $95 \%$ ) et suggère que l'avantage en taille ne suffit pas à lui seul à assurer l'implantation de l'ensemble des alevins déversés. Cette disparition peut s'expliquer par le fort taux de mortalité instantanée des individus introduits (qu'ils soient d'origine domestique ou sauvage) durant les premières semaines après leur introduction dans un nouveau milieu. A ce sujet, BERG et JÖRGENSEN (1991) ont montré dans une rivière danoise que la mortalité post-alevinage des individus $0+$ et $1+$ était plus importante les 60 premiers jours après l'alevinage que durant le reste de l'année. De même, ELLIOTT (1989) cite des taux de survie de 40 à $60 \%$ pour des alevins naturels durant les mois de mai-juin et août-septembre,

c) des déficiences de recrutement naturel vis à vis de la saturation de la capacité d'accueil en fin printemps - début été seraient comblées par le repeuplement. Ainsi les stations à fort taux de marqués en $0+$ correspondraient à des zones où le recrutement naturel est faible et les stations à faible contribution des $0+$ marqués illustreraient une bonne reproduction naturelle. Ainsi, les forts taux de $0+$ marqués observés pour la cohorte 1997 pourraient traduire un déficit de la reproduction naturelle cette année là en raison de 
conditions hydrologiques printanières défavorables à l'émergence et à l'implantation des alevins issus du recrutement naturel. Seul un protocole spécifique, relativement lourd, permettant d'étudier les variations du taux d'implantation des 0+ marqués en fonction des densités de $0+$ naturels en place permettrait de valider ou d'invalider cette hypothèse.

L'étude présente indique néanmoins que le taux d'implantation à l'automne des 0+ alevinés n'est pas corrélé avec les densités alevinées. Le taux de réussite de l'alevinage au stade $0+$ n'est donc pas fonction des quantités d'alevins déversés 4 mois plus tôt dans le milieu.

Les résultats de cette étude montrent une évolution temporelle similaire à celle obtenue sur le Doubs par DEGORGI et CHAMPIGNEULLE (2000) et conduisent donc à la formulation d'hypothèses comparables pour expliquer la dynamique observée.

En ce qui concerne le faible taux de 1+ marqués en place à l'automne, plusieurs hypothèses peuvent être émises.

a) Le taux de mortalité naturelle entre l'âge $0+$ et $1+$ qui est déjà de l'ordre de 40 à $70 \%$ pour les individus sauvages (BAGLINIĖRE et al., 1989 ; ELLIOTT, 1989), pourrait être supérieur pour les individus domestiques du fait d'une moins bonne adaptation de ces poissons au milieu torrentiel, notamment en période hivernale.

b) Une autre hypothèse pouvant expliquer la disparition des truites marquées du site d'alevinage est que les truites domestiques auraient une plus grande aptitude à la dévalaison que les individus sauvages (SKROCHOWSKA, 1969 ; CUINAT, 1971 ; GUYOMARD, 1991). Cette dévalaison touche prioritairement les truites à forte croissance (CHAMPIGNEULLE et al., 1991 ; OMBREDANE, comm. pers.) qui quittent les têtes de bassin pour rejoindre une zone de grossissement. Or CAUDRON (1998) a montré que l'avantage de taille au stade $0+$ des individus alevinés par rapport aux individus sauvages existe également au stade 1+. La présence même minime dans la zone aval du Fier d'individus $2+$ et $3+$ marqués provenant de la zone amont montre que l'hypothèse de la dévalaison n'est pas à exclure d'autant que la présence de jeunes truites smoltifiées, probablement dévalantes, est régulièrement observée par des pêcheurs au printemps dans le Fier.

c) La faible proportion de marqués d'âge supérieur ou égal à $2+$ obtenue à l'automne dans la population en place pourrait en partie s'expliquer par le fait que l'échantillonnage survient après la saison de pêche et donc ne prend pas en compte les poissons 2+ de plus grande taille déjà exploités par celle-ci.

Comme pour DEGORGI et CHAMPIGNEULLE (2000) des biais auraient pu conduire à un sous échantillonnage notamment pour les 2+ en place à l'automne en raison de la difficulté d'échantillonner par pêche électrique les habitats plus profonds particulièrement recherchés par les truites plus âgées (JONES, 1975 ; BREMSET et BERG, 1997 ; CHAMPIGNEULLE, 1978). Par contre, l'effort d'échantillonnage par pêche à la ligne se concentrerait plus sur les habitats à adultes comme les zones profondes.

d) Une dernière hypothèse consiste à penser que les individus domestiques montreraient une plus grande capturabilité par pêche à la ligne que les individus sauvages en raison d'une différence de comportement alimentaire liée à une agressivité plus importante. Des observations ont été réalisées dans ce sens en rivière artificielle par MESA (1991) sur des truites à gorge coupée (Oncorhynchus clarki). D'après CAUDRON (1998), les différences de taille entre individus domestiques et individus sauvages présentes à l'âge $0+$ et $1+$ ne sont plus présentes à partir de l'âge $2+$. En parallèle, le coefficient de condition des truites domestiques est important à l'âge 0+ quelques mois après leur introduction dans le milieu mais diminue avec le temps et à l'âge 1+ il devient 
inférieur à celui des truites sauvages. Cette observation pourrait expliquer en partie la raison d'un besoin énergétique important des individus domestiques présents en milieu naturel pour compenser leur perte pondérale.

Cette hypothèse d'une plus grande capturabilité des truites alevinées et d'une exploitation plus précoce par la pêche à la ligne ressort également de façon importante dans les études réalisées par fluoromarquage sur le Doubs franco-suisse (DEGORGI et CHAMPIGNEULLE, 2000) et par génétique sur la boucle suisse du Doubs (MEZZERA et LARGIADER, 2001).

\section{CONCLUSION ET PERSPECTIVE}

Cette étude demandée par les gestionnaires avait pour but d'estimer la contribution des repeuplements tels qu'ils étaient pratiqués sur le bassin amont du Fier c'est-à-dire avec des souches domestiques d'origine atlantique, à des stades précoces (alevins nourris et truitelles) et avec des densités faibles à moyennes (15 à 30 individus $/ 100 \mathrm{~m}^{2}$ ). Les résultats conduisent à conclure à une relative inefficacité des pratiques de repeuplement utilisées sans toutefois pouvoir préciser les causes réelles de cet échec. Cependant l'inefficacité du repeuplement ne semble pas en rapport avec les densités alevinées. De plus, l'utilisation de souche domestique atlantique dans des milieux torrentiels où les populations naturelles sont d'origine méditerranéenne est discutable.

Le résultat conduit à préconiser au minimum sur la zone d'étude une stratégie d'arrêt expérimental du repeuplement sachant que l'étude menée a montré que le risque d'effondrement de la pêcherie est minime compte tenu de la faible contribution des repeuplements pratiqués selon le mode actuel.

D'autres pratiques de repeuplement utilisant d'autres souches et d'autres stades de déversement pourraient être testées notamment sur des secteurs où un déficit de population naturelle est réellement mis en évidence. En effet, si le repeuplement dans un milieu où une population naturelle est bien implantée ne semble pas fonctionner, cela ne sera peut-être pas le cas dans un milieu naturellement déficitaire. Ainsi, l'accent devrait être mis en particulier sur le repérage du niveau de fonctionnalité des populations sur les diverses parties du réseau hydrographique du Fier. Une extension de la technique de fluoromarquage à l'ensemble des zones du bassin versant où persiste des repeuplements est une approche possible malgré l'importance de l'échelle spatiale concernée.

Enfin, il semble important de mieux connaître la composition et la diversité génétique spatiotemporelle des populations de truites (en place et pêchées) du bassin du Fier. La définition d'éventuelles nouvelles opérations de repeuplement devrait intégrer ces connaissances et résulter d'un dialogue entre gestionnaires et généticiens.

\section{REMERCIEMENTS}

Nous tenons à remercier l'ensemble de la Garderie du CSP de Haute-Savoie et la Délégation Régionale de Lyon qui ont organisé et réalisé l'ensemble des pêches électriques nécessaires à cette étude. Nous remercions également les gardes particuliers de l'AAPPMA "Annecy-Rivières » qui ont participé aux échantillonnages ainsi que les pêcheurs volontaires qui ont accepté de s'impliquer. 


\section{BIBLIOGRAPHIE}

BAGLINIÈRE J.L., MAISSE G., LE BAIL P.Y., NIHOUARN A., 1989. Population dynamics of brown trout (Salmo trutta L.) in a tributary in Brittany (France): spawning and juveniles. J. Fish Biol., 34, 97-110.

BAUMAN F., 1999. Entwicklung und Anwendung der Analyse von Konformationpolymorphismus als effiziente Methode zur Identifikation von mtDNASequenzhaplotypen bei der Forelle. Diplomarbeit der Philosophisch naturwissenschaftlichen Fakultat der Universitat Bern, 56 Seite.

BEAUDOU D., 1993. Impact des déversements de truites domestiques dans les populations naturelles de truites communes, Salmo trutta fario. Etude dynamique et génétique. Cas du bassin de l'Orb, Hérault. Thèse de doctorat, Université de Montpellier II, 308 p.

BERG S., JÖRGENSEN J., 1991. Stocking experiments with 0+ and 1+ trout parr, Salmo trutta L., of wild and hatchery origin: 1. Post-stoking mortality and smolt yield. J. Fish Biol., 39, 151-169.

BREMSET G., BERG O.K., 1997. Density, size at age, and distribution of young Atlantic salmon (Salmo salar) and brown trout (Salmo trutta) in deep river pools. Can. J. Fish. Aquat. Sci., 54, 2827-2836.

CAUDRON A., 1998. Biologie et gestion de la truite (Salmo trutta L.) d'un torrent alpin : le cas du Fier (74). Rapport de DEA Société et Environnement : gestion des espaces montagnards. Institut de Géographie Alpine, Grenoble, $65 \mathrm{p}$.

CHAMPIGNEULLE A., 1978. Caractéristiques de l'habitat piscicole et de la population de juvéniles sauvages de saumon atlantique (Salmo salar L.) sur le cours principal du Scorff (Morbihan). Thèse $3^{\text {ème }}$ cycle Biol. Anim. Fac. Sci. Univ. Rennes, $92 \mathrm{p}$.

CHAMPIGNEULLE A., MELHAOUI M., GERDEAUX D., ROJAS BELTRAN R., GILLET C., GUILLARD J., 1990. La truite commune (Salmo trutta) dans le Redon, un petit affluent du lac Léman. I. Caractéristiques de la population en place (1983-87) et premières données sur l'impact des relâchers d'alevins nourris. Bull. Fr. Pêche Piscic., 319, 181-196.

CHAMPIGNEULLE A., BUTTIKER B., DURAND P., MELHAOUI M., 1991. Principales caractéristiques de la biologie de la truite (Salmo trutta L.) dans le Léman et quelques affluents. In : " la truite : Biologie et Ecologie ». BAGLINIÈRE J.L. et MAISSE G. (Eds.), INRA, Paris, 153-182.

CHAMPIGNEULLE A., ROJAS BELTRAN R., 2001. Le marquage des poissons. In : GERDEAUX D. (ed.) Gestion piscicole des grands plans d'eau, 311-346, INRA, Paris.

CRESSWELL R.C., 1981. Post-stocking movements and recapture of hatchery - reared trout released into flowing waters - a review. J. Fish Biol., 18, 429-442.

CUINAT R., 1971. Ecologie et repeuplement des cours d'eau à truites. Bull. Fr. Piscic., 240, 242, 243.

DEGIORGI F., CHAMPIGNEULLE A., 2000. Diagnose piscicole et mesure de l'efficacité des alevinages en truite sur le Doubs Franco-Helvétique. Rapport final Etude CSPINRA, 119 p.

ELLIOTT J.M., 1989. The natural regulation of numbers and growth in conttrasting populations of brown trout, Salmo trutta, in two Lake District streams. Freswater Biology, 21, 7-19.

GUYOMARD R., 1991. Diversité génétique et gestion des populations naturelles de truite commune. In : « la truite : Biologie et Ecologie ». BAGLINIĖRE J.L. et MAISSE G. (Eds.), INRA, Paris, 215-235.

JONES A.N., 1975. A preliminary study of fish segregation in salmons spawning streams. J. Fish Biol., 7, 95-104.

MESA M.G., 1991. Variation in feeding, aggression, and position choice between hatchery and wild cutthroat trout in an artificial stream. Trans. Amer. Fish. Soc., 120, 723-727. 
MEZZERA M., LARGIADER C., 2001. Evidence for selective angling of introduced trout and their hybrids in a stocked brown trout population. J. Fish Biol., 59, 287-301.

OMBREDANE D., RICHARD A., 1990. Détermination de la zone optimale de prélèvement d'écailles chez les smolts de truite de mer (Salmo trutta L.). Bull. Fr. Pêche Piscic., 319, 224-238.

POTEAUX C., BERREBI P., 1997. Intégrité génomique et repeuplement chez la truite commune du versant méditerranéen. Bull. Fr. Pêche Piscic., 344/345, 309-322.

ROJAS BELTRAN R., CHAMPIGNEULLE A., VINCENT G., 1995a. Mass-marking of bone tissue of Coregonus lavaretus $\mathrm{L}$. and its potential application to monitoring the spatio-temporal distribution of larvae, fry and juveniles of lacustrine fishes. Hydrobiologia, 300/301, 399-407.

ROJAS BELTRAN R., GILLET C., CHAMPIGNEULLE A., 1995b. Immersion mass-marking of otoliths and bone tissue of embryos, yolk-sac fry fingerligs of artic charr Salvelinus alpinus L. Nordic Journal of Freshwater Research, 71, 411-418.

SKROCHOWSKA S., 1969. Migrations of the sea trout (Salmo trutta), brown trout, and their crosses. Pol. Arch. Hydrobiol., 16, 125-192.

SPRENT P., 1992. Pratique des statistiques non paramétriques. INRA (Eds), Paris, $294 \mathrm{p}$.

THIBAULT M., 1983. Les transplantations de Salmonidés d'eau courante en France, saumon atlantique (Salmo salar L.) et truite comune (Salmo trutta L.). C.R. Soc. Biogeogr., 59, 405-420.

TSUKAMOTO K., KUWADA H., HIROKAWA J., OYA M., SEVIKA S., FUJIMOTO H., IMAIZUMI K., 1989. Size-dependent mortality of red sea-bream (Pagrus major) juveniles released with fluorescent otolith-tags in New-Bay, Japan. J. Fish Biol., 35, 59-69.

WILLIAM H., BEYER P.D., 1986. CRC Hanbook of Tables for Probability and Statistics. CRC Press, Inc. Boca Raton, Florida. 
